\title{
Mixoma Gigante de Átrio Esquerdo
}

\section{Juan Francisco Muñoz Cruz, Leniel Bairral Dias, João Osório Rodrigues Neto, Sérgio Almeida de Oliveira}

\author{
Volta Redonda, RJ - São Paulo, SP
}

Homem de 65 anos, portador de miocardiopatia dilatada e hipertensão arterial de longa data, com antecedentes de acidente vascular cerebral e que, ao ecocardiograma, apresentou mixoma gigante de átrio esquerdo.

\section{Giant Left Atrial Myxoma}

A 65 year-old man with dilated cardiomiopathy and long history of high blood pressure and a previous cerebral vascular accident had a giant left atrial myxoma as an echocardiographic finding.

Arq Bras Cardiol, volume $71\left(n^{0}\right.$ 5), 717-718, 1998

Mixoma é o mais freqüente tumor cardíaco primário ${ }^{1}$. Em cerca de $90 \%$ dos casos localiza-se no átrio esquerdo (AE), é pediculado e, habitualmente, preso à fossa oval. Entre as freqüentes complicações, são encontrados os fenômenos embólicos. Descrevemos um caso de paciente oligossintomático com tumor de longa duração.

\section{Relato do Caso}

Homem de 65 anos, natural de Rio Preto (MG), hipertenso em tratamento há cerca de 15 anos, com queixas de dispnéia e dor torácica aos esforços, desde o início do tratamento. Seus exames no início revelaram área cardíaca aumentada $++/ 4$ do ventrículo esquerdo (VE) e bloqueio do ramo direito ao eletrocardiograma. Na época apresentava turgência jugular importante, hepatomegalia e ausculta cardíaca normal. Acometido de dois episódios de acidente vascular cerebral, há 10 e seis anos. Houve agravamento dos sintomas, há cerca de três anos, quando foi notado desenvolvimento de fibrilação atrial crônica. Realizado Doppler ecocardiograma que revelou miocardiopatia dilatada com moderada disfunção sistólica do VE, $\% \Delta \mathrm{D}$ de $25 \%$ e fração de ejeção de $43 \%$, grande aumento atrial esquerdo, doença degenerativa valvar mitro-aórtica e grande mixoma pediculado em $\mathrm{AE}$, com cerca de $6,5 \mathrm{~cm}$ de diâmetro, praticamente imóvel, e permitindo diástole satisfatória, insuficiên-

Clínica Cardiológica Dr Leniel Bairral Dias, Volta Redonda e Real e Benemérita Beneficência Portuguesa de São Paulo

Endereço: Juan Francisco Muñoz Cruz - Rua Thomas Antônio Gonzaga, 69/403 27250-530 - Volta Redonda, RJ

Recebido para publicação em 24/6/98

Aceito em 9/9/98 cia mitral e aórtica leves (fig. 1 e 2). Estudo hemodinâmico revelou lesão de cerca de 50\% em terço médio da artéria descendente anterior, elevação leve das pressões em câmaras direitas, insuficiência mitral discreta e mixoma pediculado em AE, VEdilatado e com hipocinesia difusa ++/4 (fig. 3).

Na cirurgia, foi realizada exérese do mixoma que pesava cerca de $85 \mathrm{~g}$, diâmetro de $6,5 \mathrm{~cm}$, superfície lisa e, ao exame microscópico, revelou neoplasia mesenquimal madura, caracterizada por proliferação de células globosas, com citoplasma acidófilo e núcleos picnóticos. As células estavam isoladas, às vezes formando cordões em meio a ampla matriz mixóide, focos de hemorragia e siderófagos, firmando o diagnóstico de mixoma com hemorragia.

A cirurgia e o pós-operatório transcorreram sem intercorrências e o paciente recebeu alta no $8^{\circ}$ dia de pósoperatório em uso de ácido acetilsalicílico e diurético.

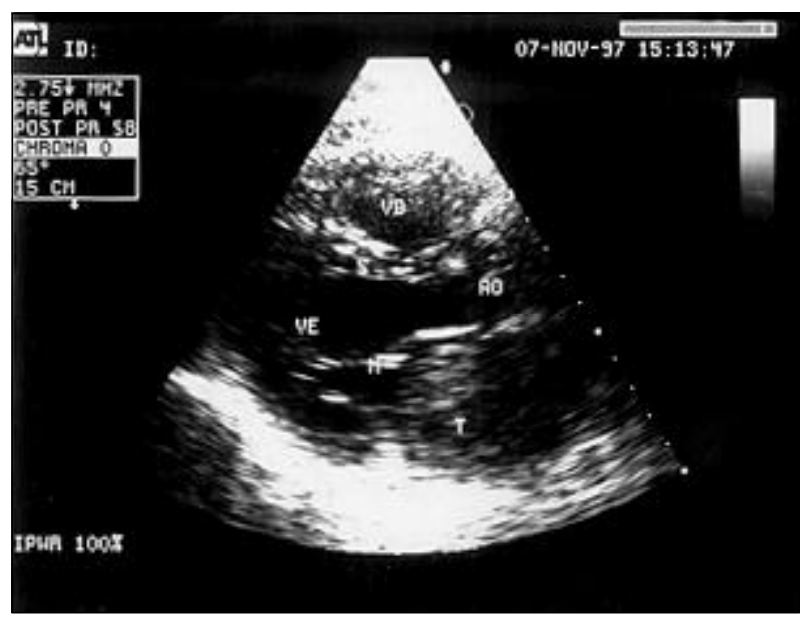

Fig. 1 - Ecocardiograma em corte paraesternal longitudinal visibilizando o mixoma em átrio esquerdo. 


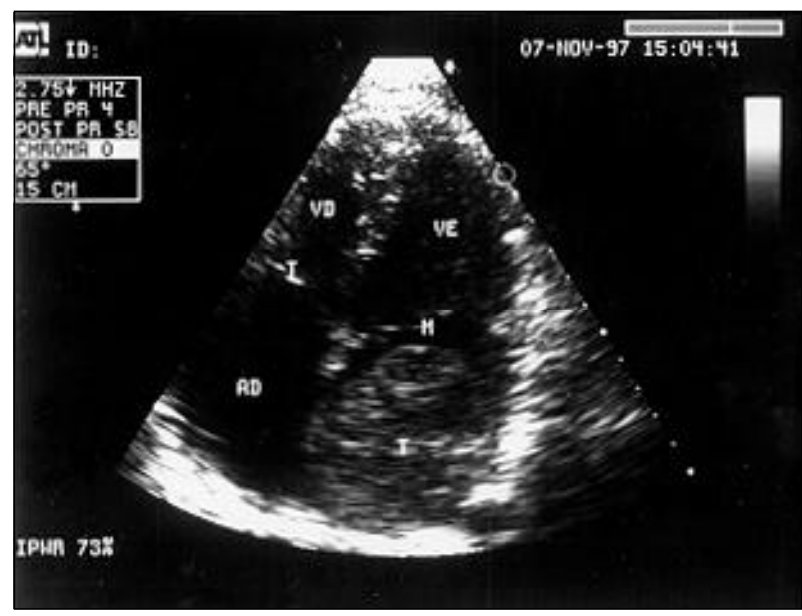

Fig. 2 - Ecocardiograma corte apical quatro câmaras visibilizando dilatação de cavidades esquerdas e mixoma gigante em átrio esquerdo.

\section{Discussão}

Entre os tumores primários do coração, os mixomas são os mais freqüentes e representam cerca de $50 \%$ dos ca$\operatorname{sos}^{1}$. Quando localizados no AE, representam $90 \%$ casos e é mais freqüente em mulheres ( $76 \%$ casos $)^{2-4}$ e na $5^{\text {a década. }}$ Em indivíduos com mixomas familiares, notamos que a exteriorização do quadro inicia-se por volta da $2^{\mathrm{a}}$ à $4^{\mathrm{a}}$ décadas, geralmente, com síncopes ou embolizações sistêmicas.

Os mixomas esporádicos têm evolução variável e exteriorizam-se, habitualmente, sob a forma de sintomas decorrentes de uma possível valvopatia mitral (43\% dos casos), embolias sistêmicas (27\%), embolias pulmonares (25\%), morte súbita $(15 \%)$, pericardite $(0,03 \%)$, fibrilação atrial (15\%) e totalmente assintomáticos em $12 \%$.

No nosso relato, encontramos um portador de miocardiopatia dilatada, com provável fundo hipertensivo, oligossintomático, que apresentou, no decorrer de 15 anos, dois episódios de acidente vascular cerebral embólico, com provável etiologia do mixoma. Como já foi descrito, tumores com superfície lisa, encapsulados, têm

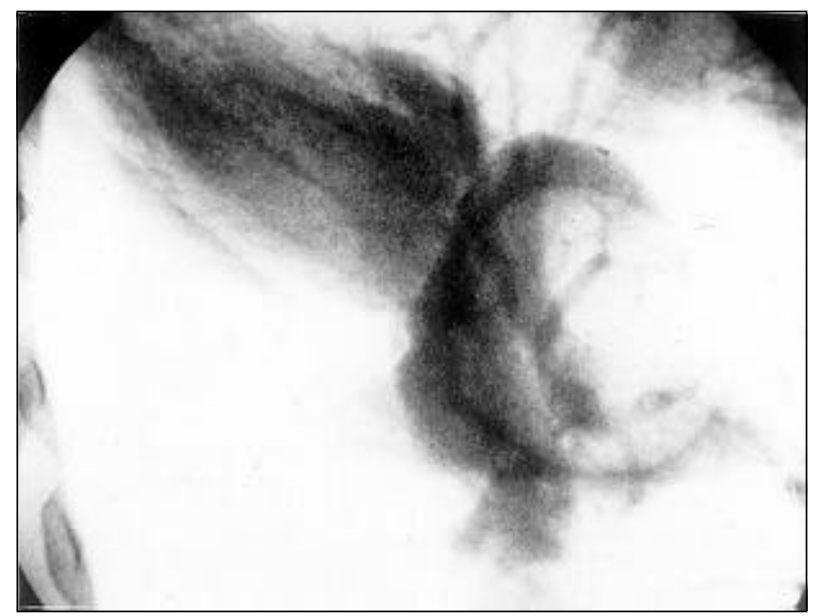

Fig. 3 - Estudo hemodinâmico revelando levograma em OAD volumoso mixoma pediculado em átrio esquerdo.

menor probabilidade de embolização, seja ela sistêmica ou coronária, sendo esta muito rara e com incidência por necropsias em torno de $0,06 \%{ }^{4}$.

Êmbolos sistêmicos múltiplos podem nos fazer pensar em diversas outras doenças, como vasculites sistêmicas, endocardite infecciosa e trombos intracavitários. Neste presente caso houve suspeita inicial de trombose ventricular esquerda, face à doença de base.

A sintomatologia é bastante variada e com mais frequiência vemos dispnéia aos esforços, dispnéia paroxística, febre, emagrecimento, síncopes e pré-síncopes, hemoptises e até morte súbita. Entre os sinais mais freqüentes, encontramos sopros diastólicos e sistólicos mitrais, hipertensão pulmonar e insuficiência cardíaca direita.

No nosso caso, a pobreza de sinais e a sintomatologia decorrente da insuficiência cardíaca esquerda fizeram com que não se chegasse ao diagnóstico clínico.

Concluímos, portanto, que na cardiologia moderna, uma completa anamnese, um correto exame físico e a moderna tecnologia dos equipamentos, conseguem sempre um diagnóstico preciso e precoce ${ }^{6}$.

\section{Referências}

1. Lammer RJ, Bloor CM - Pathology of cardiac tumors. In: Kapoor AS (ed) - Cancer of the Heart. New York: Springer-Verlag, 1986.

2. Carvalho RG, Giublin PR, Rachid A, Costa IA, Cunha GP- Mixoma de átrio esquerdo. Aspectos cirúrgicos e ultra-estruturais. Arq Bras Cardiol 1991; 51: 171-5.

3. Carney JA - Differences between nonfamilial and familial cardiac myxoma. Am J Pathol 1985; 9: 53.
4. Meira EBS, Liang Ho C, Santos Filho MM, Pimenta J - Mixoma de átrio esquerdo provocando infarto agudo do miocárdio. Arq Bras Cardiol 1996; 67: 347-9.

5. Braunwald E - Tratado de Medicina Cardiovascular. Cap. 43, vol. 2. São Paulo: Ed Roca, 1991: 1538-50.

6. Weitzel LH - Tumores cardíacos. Avaliação por ecocardiografia. ARS Curandi 1986; 63: 47. 